\title{
Optical properties of dense self-assembled gold nanoparticle layers with organic linker molecules
}

\author{
S. Schelm ${ }^{a}$. G. B. Smith ${ }^{a}$. G. Wei ${ }^{b}$. A. Vella ${ }^{a, b}$, L. Wieczorek ${ }^{b}$, K.-H. Müller ${ }^{b}$ and B. Raguse ${ }^{b}$ \\ aDepartment of Applied Physics. University of Technology. Sydney, PO Box 123 Broadway. \\ NSW 2007, Sydney, Australia \\ ${ }^{b}$ CSIRO Telecommunications \& Industrial Physics, PO Box 218 Lindfield, NSW 2070, Sydney. \\ Australia
}

\begin{abstract}
Films consisting of self-assembled gold nanoparticles cross-linked with alkane-dithiols were prepared by a filtration method and studied with scanning electron microscopy to determine the structure of the films and spectrophotometry and ellipsometry to ascertain their optical properties. The structural characterization showed the existence of nanometer-sized voids within the films. This previously unmentioned feature is responsible for the previous difficulties in modelling the optical properties with effective medium models. This can be remedied. using a two-tiered hierarchical effective medium model, which takes into account the existence of the voids. Using this model we were able to fit the experimental data, with only the void volume fraction to be determined by the overall fit, while the gold volume fraction in the linker medium is fixed by the wavelength of the resonance peak. Our model should be applicable to all such films, when the deposition method, which determines the microstructure. is properly taken into account.
\end{abstract}

Keywords: gold. dithiol. self-assembled. optical properties, effective medium approximation

\section{INTRODUCTION}

Thiol covered gold surfaces have attracted a wide interest for some time. Wore recently: that interest has spread to the area of thiol-encapsulated gold nanoparticle (NP) films (for an overview. see Ref. 1 and References therein). Both electrical ${ }^{1-4}$ and optical properties ${ }^{1.4,5}$ have been studied, but a general model for the different optical data reported has not been available yet. Although individual publications tried different models to fit their data. they either did not find a model that worked ${ }^{5}$ or the model was oversimplified and neglected particle interaction and the actual nanostructure. ${ }^{4}$ We propose, for the first time. a model that can describe all of the reported optical data. The important point which has not been recognized until now. is the role nanometer-sized voids are plaving in the films. Scanning electron microscopy (SEM) characterization of our films with a new zeneration Schottky field emission gun SEM has enabled us to examine the microstructure of the films with a yood enough resolution to reveal the nano-sized voids. Based on these findings. we propose a double effective medium approximation (EMIA) model to describe the optical properties. The parameters which enter this model ure the dielectric functions of the gold NPs and thiol (which might differ for different deposition techniques) and the fill factors $f_{A u L}$ and $f_{\text {rold }}$. where $f_{A u L}$ is the volume fraction of gold in the linker medium and $f_{\text {void }}$ is the :olume fraction of the voids within the whole film system. The remaining parameters are the thickness of the sims and the microstructure of the roid inclusions (expressed in the choice of the specific EllA form).

\section{SYNTHESIS OF FILMS}

The preparation of our nanoparticle films has been described previously by Raguse et al. ${ }^{6}$ but we will briefly sutline the procedure here. Toluene suspensions of gold nanoparticles with an average size of $6 \mathrm{~nm}$ and coated zith tetraoctylammonium bromide were prepared by the method of Brust et al..$^{7 \cdot 3}$ All chemicals were purchased from Sigma-Aldrich Pty: Ltd. and used without further purification. Isopore membrane filters ( $200 \mathrm{~nm}$ pore size)

\footnotetext{
Send correspondence to:

E-mail: stefan.schelm @uts.edu.au
} 


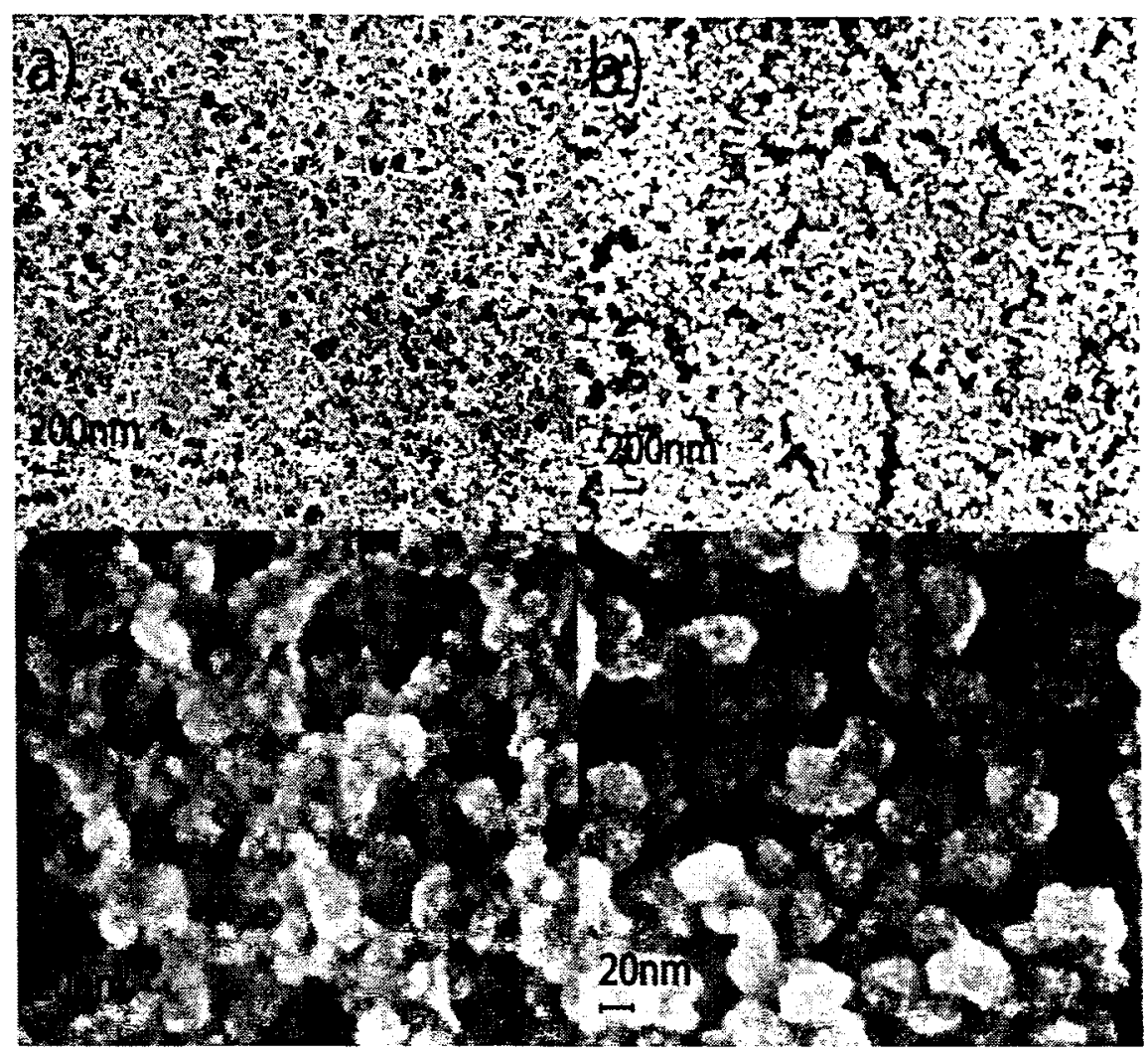

Figure 1. SEM images (accel. voltage $12 \mathrm{kl}^{\circ}$ ) for films produced from $3.0 \mathrm{ml}$ of solution for the two linker molecules studied: a) C8-dithiol. b) C15-dithiol

were obtained from Millipore Corporation and Anodisc membrane filters (20 nm pore size) were obtained from Whatman International Ltd. The nanoparticles were cross-linked by adding a solution of either 1.8-octanedithiol (C8-dithiol) or 1,15-pentadecanedithiol (C15-dithiol) ( $10 \mu \mathrm{l} .0 .1 \mathrm{M}$ in toluene) to $10 \mathrm{ml}$ of freshly prepared $6 \mathrm{~nm}$ gold nanoparticle suspension. The resultant mixture was swirled for a while and set aside for up to 15 minutes and diluted with an equal part of n-hexane. then racuum-filtered through the substrate (Isopore or Anodisc membrane filters), followed by rinsing with n-hexane. The gold nanoparticle films thus formed on the surface of the substrate, with a size of $3 \mathrm{~cm}^{2}$. The films were air-dried at least overnight before any characterization was conducted. Isopore membranes trere used for the optical studies. and porous alumina (Anodisc) membranes wrere used for cross section analrsis. Films of different thickness were made by filtrating different volumes of cross-linked gold nanoparticle suspensions. The thimner filns appeared grevish in color. whereas nanoparticle films prepared from $3 \mathrm{ml}$ of solution and more appeared metallic golden.

\section{SEM CHARACTERIZATION OF FILMS}

Fig. $1 \mathrm{a}$ and b show SEM images taken with a LEO 1550-YP Schottly field emission gun SEM. of the microstructure of two films, filtrated from the same amount of solution. for the two linker molecules used: C8-dithiol and C15-dithiol, respectively. The iniages reveal a three phase sustem. consisting of gold XPs. thiol molecules and voids. These voids are of importance, as ther affect the description of the optical properties with an EMIA model. Without the proper consideration of these voids in an E.IA. the model is inconsistent with the actual nanostructure and thus camnot describe the data fully:

The bright spots, which can be seen in the high magnification inages. are the actual gold XPs. while the slightly darker area around them corresponds to the linker molecules. One important finding is that the particles seem to be separated from each other. Any apparent coilescence in the images is due to the three dimensionality 


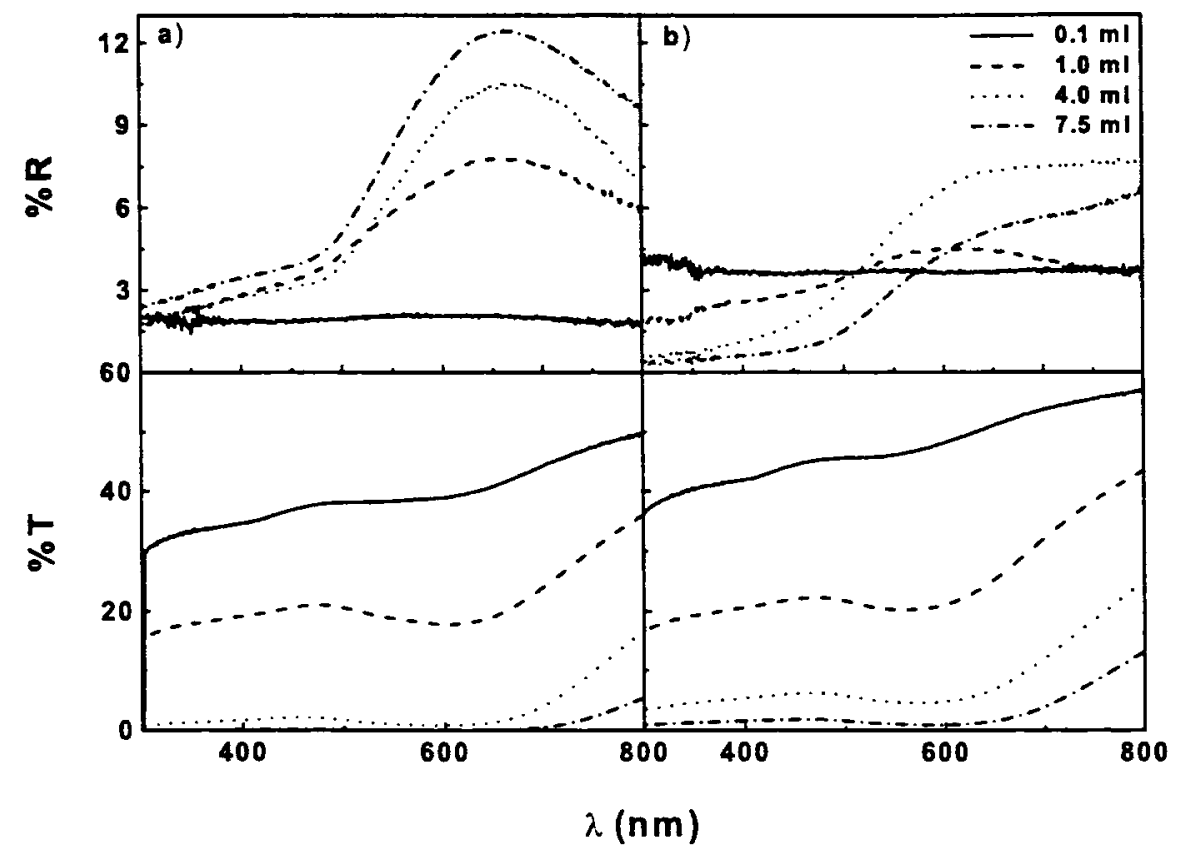

Figure 2. Reflectance and Transmittance for films produced from different amounts of solution (refer to Table 1 for the corresponding thicknesses) for the two linkers studied: a) C8-dithiol, b) C15-dithiol

of the films. creating superpositions in the images of particles at different depths. This is verified by the fact that our optical model is sensitive to the issue of particle aggregation and no indication of this could be found in our analysis. There is a difference in the microstructure between the two linker molecules used. While the shorter linker (C8-dithiol) builds a network of thin wire-like structures, the building blocks of the longer linker (C15-dithiol) seem to be roughly spherical globules sticking together.

IVe also used the SEM to estimate the thickness of the films from their cross-sections. The cross-sections were prepared on a porous alumina substrate. Assuming a linear increase in the film thickness with respect to the filtrated volume, we extrapolated to determine the thickness of the films for which no cross-sections were available. Judging from the cross-section images. the structure does not change much across the film length and depth. The results for the thinner films have to be treated more carefully, as these films are not continuous but have a rather island-like structure, as verified by SEM. A more comprehensive investigation of the different micro-structures will be published elsewhere. ${ }^{9}$

\section{OPTICAL CHARACTERIZATION OF FILMS}

The optical properties of the films have been determined by spectrophotometry and ellipsometry. Reflectance and transmittance results for exemplary films are shown in Fig. 2 and the real and imaginary parts of the refractive index. $\mathrm{n}$ and $\mathrm{k}$ derived from ellipsometry (angle of incidence $=70^{\circ}$ ). are shown in Fig. 3. The transmittance data of Fig. 2 show a very broad and weak absorption. centered at around $610 \mathrm{~nm}$. The ellipsometry measurements in Fig. 3 show that although the thicker films look golden. this is not due to a bulk-like response. as can be seen from the comparison with the inset. which shows bulk data from Ref. 10. In bulk gold $\mathrm{k}$ increases for wavelengths larger than $500 \mathrm{~nm}$. while $\mathrm{n}$ decreases in the same region. The real part of the refractive index. $\mathrm{n}$. of our films actually reverses the trend from the bulk dara. This show's that these films do not show a normal metallic response. The gold-like appearance is rather due to the position of the surface plasmon at around $610 \mathrm{~nm}$. which in turn clepends on the particle material. the host medium and the volume fraction of the particles.

Another point which we derived from our measurements is that the films can be organized in two regimes. with respect to the coverage of the deposited material onto the filter membranes: 1) an island-like regime. for films made from small volumes of solution and 2) a continuous region. for films from larger volumes. Apart from 

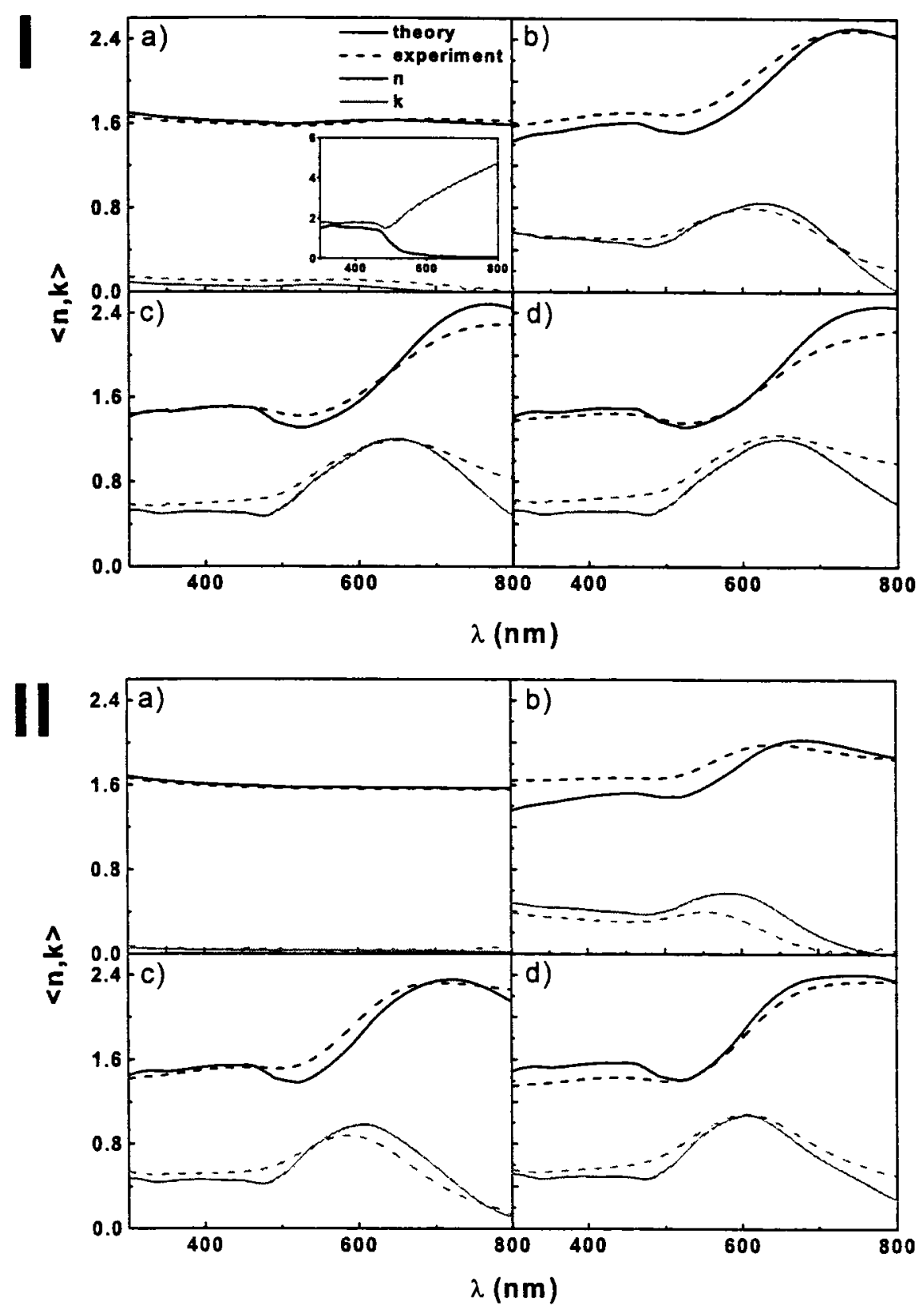

Figure 3. Effective $\mathrm{n}$ and $\mathrm{k}$ values for different films on the isopore substrates, as measured with ellipsometry and calculated results from our double EMA model (with parameters from Table 1), for the two linker molecules: I) C8-dithiol (the inset shows the bulk data from Ref. 10 ). II) C15-dithiol and films produced from the amounts of solution shown in Fig. 2: a) $0.1 \mathrm{ml}$. b) $1.0 \mathrm{ml} . \mathrm{c}) 4.0 \mathrm{ml}$ and d) $7.5 \mathrm{ml}$ 
SEM characterizations, which support this assumption, the optical properties themselves show evidence for two regimes. If the continuous phase in our samples can be treated with one effective dielectric function. as we will show in the next section. one would expect a transition from thin island or semi-continuous films to thicker continuous ones. These continuous films should have a constant optical response. independent of the actual film thickness. This is exactly what we observed in our ellipsometry measurements. with the transition thickness corresponding to a filtrated volume of $2 \mathrm{ml}$ (see Tab. 1 for an over iew).

\section{OPTICAL MODEL: DOUBLE EMA APPROACH}

The major point of this paper is the proposition of a double EMA approach to explain the optical properties of the nano-gold-thiol srstem. We explain this with the hierarchical structure of the films. which can be seen in the SEM images of Fig. 1. The first tier of the hierarchy is created by the gold-thiol system. As most of our SEMI images show a clear separation between the randomly arranged gold particles. as well as the complete encapsulation of the gold by the thiol. we can treat this first composite component with the Maxwell-Garnett (MG) EMA. ${ }^{11}$ This component describes the isolated gold NPs in the dithiol linker medium. rielding the dielectric function for the first composite $\Xi_{A} \mathrm{AL}$. according to the following formula for spherical inclusion: ${ }^{12}$

$$
\frac{\varepsilon_{A u L}-\varepsilon_{L}}{\varepsilon_{A u L}+2 \varepsilon_{L}}=f_{A u L} \frac{\varepsilon_{A u}-\varepsilon_{L}}{\varepsilon_{A u}+2 \varepsilon_{L}} \text {. }
$$

where $\Sigma_{L}$ and $\Xi_{A u}$ are the dielectric functions of the linker and the gold nano-particles, respectively: The Drude part of the dielectric function for the gold was size corrected ${ }^{13}$ using half $\left(\mathrm{d}_{\epsilon f f}=3 \mathrm{~nm}\right)$ the physical size of the particles and experimental bulk data from Ref. 10. This reduction was necessary to broaden the resonance to an extent found in the samples and is not unreasonable as other effects might further decrease the electron relaxation time (e.g. grain boundaries and chemical interface effects). ${ }^{13.14}$ This effective size reduction, with the same values (phrsical size: $6 \mathrm{~nm}$. effective size: $3 \mathrm{~nm}$ ). was also used in Ref. 4 to improve the fit to their data.

The second tier is made by including voids into the gold-thiol MG E.IA. A schematic of this model can be seen

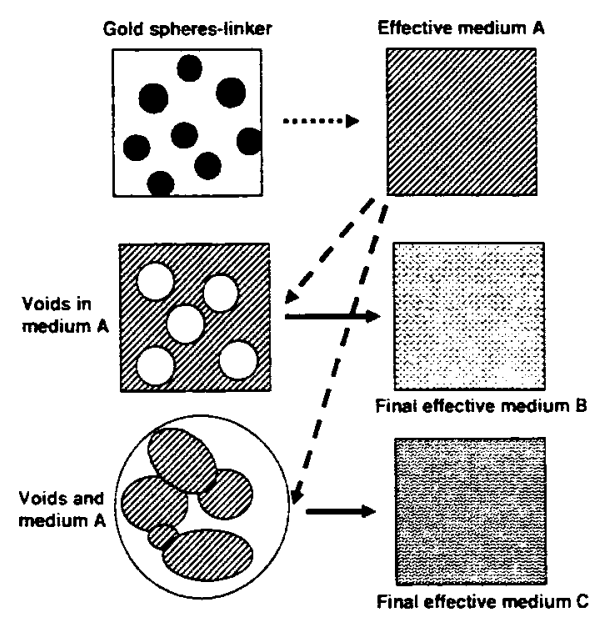

Figure 4. Schematics of our double ENA for the inclusion of the voids either in MG EMA (final effective medium B) or Bruggeman E.MA (final effective medium C)

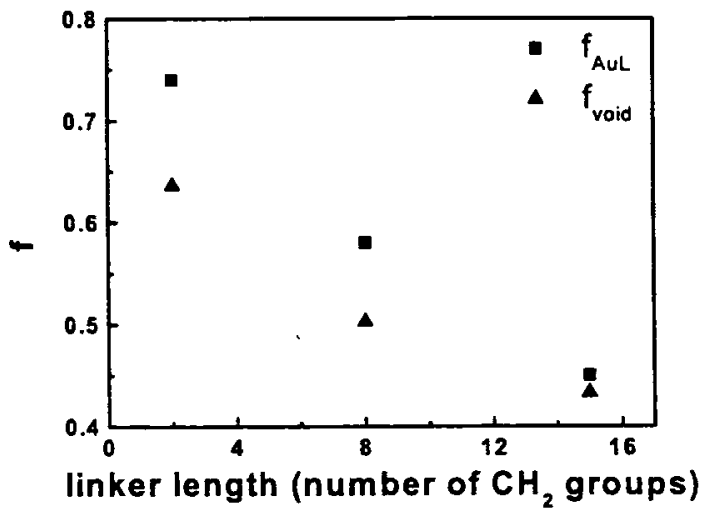

Figure 5. Dependence of the volume fractions $f_{A u L}$ and $f_{\text {rord }}$ on the linker length (this graph includes tentative preliminary results for the C2-dithiol linker)

in Fig. 4. The choice of the specific model in the second tier is not as clear as in the first one. but based on the percolated structure the voids show in the SEM images. we chose to include the voids within the Bruggeman EMA model. To confirm this choice. we compared MG and Bruggeman for the void inclusion. We found that the 
Table 1. Thicknesses and void fractions for the films produced with the two different cross linkers $\left(f_{A u L}\right.$ derived from the $\mathrm{k}$ peak position is 0.58 and 0.45 for $\mathrm{C} 8$-dithiol and $\mathrm{C} 15$-dithiol, respectively)(the starred values represent actually. measured cross-sections)

\begin{tabular}{|c|c|c|c|c|}
\hline filtrated volume (ml) & $\begin{array}{c}\text { C8-dithiol } \\
\text { thickness (nm) }\end{array}$ & $\mathrm{f}_{\text {voids }}$ & $\begin{array}{c}\text { C15-dithiol } \\
\text { thickness (nm) }\end{array}$ & $\mathrm{f}_{\text {voids }}$ \\
\hline 0.1 & 5 & 0.715 & 6 & 0.862 \\
\hline 0.2 & 10 & 0.701 & 11 & 0.854 \\
\hline 0.5 & 24 & 0.613 & 27 & 0.674 \\
\hline 1.0 & 48 & 0.584 & 55 & 0.611 \\
\hline 2.0 & $96^{*}$ & 0.506 & $109 *$ & 0.386 \\
\hline 3.0 & 144 & 0.491 & 164 & 0.422 \\
\hline 4.0 & 193 & 0.504 & 219 & 0.442 \\
\hline 5.0 & 241 & 0.503 & 273 & 0.456 \\
\hline 7.5 & $360^{*}$ & 0.505 & $410^{*}$ & 0.405 \\
\hline 10.0 & 481 & 0.514 & 547 & 0.441 \\
\hline
\end{tabular}

Bruggeman model works better for almost all films, with only the three thickest C15-dithiol films as exceptions. where the MG gires slightly better results. Even though, the difference is not big enough to force the use of MG and the Bruggeman was used for all films. The equation for the Bruggeman EMA model for spherical inclusions is: ${ }^{12}$

$$
\left(1-f_{\text {void }} \frac{\Xi_{A u L}-\Xi^{*}}{\Xi_{A u L}+2 \varepsilon^{*}}+f_{\text {void }} \frac{1-\varepsilon^{*}}{1+2 \varepsilon^{*}}=0 .\right.
$$

where $f_{\text {void }}$ is the roid volume fraction. $\hat{\varepsilon}_{A u L}$ as derived from Eq. 1 and $\varepsilon^{*}$ the dielectric function of the complete system, representing the whole film. We attribute the deviation of the thick $\mathrm{C} 15$-dithiol films from the beharior of all other films to the increased isolation of the roids in these films. This situation would have to be treated in the MG EMA. ${ }^{11}$ That the difference between the two models is not large. is an indication that this is a transition region. where the difference in the microstructure does not demand a specific EMA model. The final step of the model was to include the double EXIA model into the Woollam multilaver program IITase32. on top of a $10 \mu \mathrm{m}$ polycarbonate substrate.

Three parameters are to be determined within our model: the volume fractions of the gold in the linker medium. $f_{A u L}$, the volume fraction of the voids in the complete gold-linker-void system, $f_{\text {roid }}$ and the thickness of the layer. The spectral position of the resonance peak in $k$ is very sensitive to $\mathrm{f}_{A u L}$. By comparing modelled and experimental peak, we were able to determine $\mathrm{f}_{A u L}$ within an error of 0.01 , with $\mathrm{f}_{A u L}$ values of 0.58 and 0.45 for C8-dithiol and C15-dithiol, respectively. For the thickness of the double EMA layer, we used the results from the SEM cross-section analysis. Therefore the only parameter determined by the fit was $\mathrm{f}_{\text {void }}$. The results for the thicknesses and $\mathrm{f}_{\text {void }}$ for the studied linkers are summarized in Table 1. As can be seen from this table. the continuous regime, in which we expect thickness independent optical properties, is reached with a filtrated volume of approximately $2 \mathrm{ml}$. This is supported by the optical data for the films made from more than $2 \mathrm{ml}$. of which we have shown examples in this paper. The general observed trend of decreasing void fraction with increasing film thickness is supported from our SEM characterization. which showed that the two thickest films for each linker are becoming. at least at the surface. more compact. The dependence of the volume fractions $f_{A u L}$ and $f_{\text {roid }}$ on dithiol length is shown in Fig. 5. This figure includes tentative preliminary results for a third linker length studied (C2-dithiol) and $\mathrm{f}_{\mathrm{r} \text { oid }}$ is calculated as the average from the individual films in the continuous regime (the relative error in taking the films from $2 \mathrm{ml}$ to $10 \mathrm{ml}$ is below $1 \%$ in the case of C8-dithiol and below $3 \%$ for the $\mathrm{C} 15$-dithiol). The graph shows that $\mathrm{f}_{4 u L}$ decreases with increasing linker length. This is just as expected because the separation between the particles is increasing. It looks almost like a linear behavior. though three points are too fer to be certain. $\mathrm{f}_{\text {zoid }}$ is also decreasing with increasing linker length. though this time it is not a linear decrease. This behavior cannot be explained as simply as that for $\mathrm{f}_{A u L}$. but it must be due to the microstructure of the films. which is controlled by the time allowed for cross-linking and the filtration process details. Judging from the SEM results for the two linkers studied, we think that the films with longer 
molecules get more compact and develop more round clusters. They are therefore less porous and have a lower void fraction. If even longer dithiols would follow the nonlinear trend, it would indicate a saturation limit. which seems to level of at around $40 \%$ of voids. This might be explained in the future by fundamental interactions between the dithiol molecules and the gold NPs.

\section{CONCLUSION}

We presented a hierarchical EMA model to explain the wide range of optical properties reported for films of gold nanoparticles cross-linked with alkane-dithiol molecules, prepared via a filtration process. This model includes a feature which has not been recognized before. which is the role nanometer-sized voids play in the films. This feature was found while examining our samples with SEM. The proper treatment of these voids within our double EMA model enabled us to explain the optical properties. which were determined by spectrophotometry and ellipsometry. The combined optical and structural characterization used here seems of special importance in these kinds of films due to the creation of unique microstructures. which have to be considered in optical models. We are certain that the optical data previously reported can be explained within the framework of our model. if the specifics of the deposition methods used (e.g. a reduced $\varepsilon_{L}$ from a less dense linker medium would explain the blue-shift of the resonance reported in Ref. 5) are properly accounted for. Our results also show that the deposition technique is critical in the development of the optical response.

\section{ACKNOWLEDGMENTS}

Stefan Schelm wants to acknowledge the support from BASF AG. Germany, for support of his PhD. We also would like to express our thanks to Dr. Richard Wuhrer for much help with the SEMI characterization.

\section{REFERENCES}

1. M. Brust. D. Bethell. C. J. Kiely. and D. J. Schiffrin. "Self-assembled gold nanoparticle thin films with nonmetallic optical and electronic properties," Langmuir 14(19). pp. 5425-5429. 1998.

2. P. E. Trudeau, A. Orozco. E. Kran. and A. A. Dhirani. "Competitive transport and percolation in disordered arrays of molecularly-linked au nanoparticles." J. Chem. Phys. 117(8). pp. 39iz-3981. 2002.

3. K.-H. Müller. J. Herrmann. B. Raguse. G. Baxter. and T. Reda. "Percolation model for electron conduction in films of metal nanoparticles linked by organic molecules," Phys. Rev. B 66( $(\overline{)})$. pp. art. no.-075417. 2002.

4. T. Baum. D. Bethell. X. Brust. and D. J. Schiffrin, "Electrochemical charge injection into immobilized nanosized gold particle ensembles: Potential modulated transmission and reflectance spectroscopy:" Langmuir 15(3). pp. 866-8i1. 1999.

5. H.-L. Zhang. S. D. Evans. and J. R. Henderson. "Spectroscopic ellipsometric evaluation of gold nanoparticle thin films fabricated using layer-by-layer self-assembly." Adv. Mater. 15(6). pp. 531-534. 2003.

6. B. Raguse, J. Herrmann. G. Stevens. J. Myers. G. Baxter, K.-H. Müller. T. Reda. A. Molodyk. and V. Braach-Maksvytis. "Hybrid nanoparticle film material." J. Nanopart. Res. 4. pp. 137-143, 2002.

7. M. Brust. M. Walker. D. Bethell. D. J. Schiffrin. and R. Whyman. "Srnthesis of thiol-derivatized gold nanoparticles in a 2-phase liquid-liquid svstem." J. Chem. Soc.-Chem. Commun. ( 7 ). pp. 801-802. 1994

8. M. Brust. D. Bethell. D. J. Schiffrin. and C. J. Kiely; "Novel gold-dithiol nano-netrorks with nonmetallic electronic- properties." Adv. Mater. 7(9). p. 795. 1995.

9. S. Schelm et al.. to be published.

10. J. H. Weaver. C. Krafka. D. W. Lynch. and E. E. Koch. Optical Properties of Metals. Part II. Physics Data No. 18-2. Fachinformationszentrum Energie. Physik. Mathematik. Karlsruhe. 1981.

11. G. Smith. "Dielectric constants for mixed media." J. Phys. D-Appl. Phys. 10(4). pp. L39-42. 1977.

12. G. A. Niklasson. "Optical properties of inhomogeneous two-component materials." in Materials Science for Solar Energy Conversion Systems. C. Granquist. ed.. Renewable Energy. pp. $`-43$. Pergamon Press. Oxford. 1st ed.. 1991.

13. U. Kreibig and M. Vollmer. Optical properties of Mctal Clusters. Springer Series in Materials Science. Springer-Verlag Berlin Heidelberg New York. 1995.

14. B. X. J. Persson. "Polarizability of small spherical metal particles: influence of the matrix environment." Surf. Sci. 281(1-2). pp. 153-162. 1993. 